\title{
Circuit
}

Musiques contemporaines

\section{Henri Pousseur : Couleurs croisées et La seconde apothéose de Rameau, Orchestre philharmonique de Liège et ensemble Musiques Nouvelles, dirigés par Pierre Bartholomée}

\section{Michel Gonneville}

Volume 15, numéro 2, 2005

Cartes d'identités

URI : https://id.erudit.org/iderudit/902361ar

DOI : https://doi.org/10.7202/902361ar

Aller au sommaire du numéro

Éditeur(s)

Les Presses de l'Université de Montréal

ISSN

1183-1693 (imprimé)

1488-9692 (numérique)

Découvrir la revue

Citer ce compte rendu

Gonneville, M. (2005). Compte rendu de [Henri Pousseur : Couleurs croisées et La seconde apothéose de Rameau, Orchestre philharmonique de Liège et ensemble Musiques Nouvelles, dirigés par Pierre Bartholomée]. Circuit, 15(2), 101-104. https://doi.org/10.7202/902361ar d'utilisation que vous pouvez consulter en ligne.

https://apropos.erudit.org/fr/usagers/politique-dutilisation/ 


\section{Chroniques}

Henri Pousseur : Couleurs croisées et La seconde apothéose de Rameau, Orchestre philharmonique de Liège et ensemble Musiques Nouvelles, dirigés par Pierre Bartholomée

Quel plaisir que de trouver ici réunies deux œuvres-phares du compositeur belge Henri Pousseur, et dans quelles magistrales interprétations!

La première œuvre témoigne de l'efficacité expressive de la théorie harmonique du compositeur lorsque celle-ci est mise en œuvre. Elle est aussi un exemple de musique "réaliste ", pourrait-on dire à la suite de Michel Butor, ( «La musique, art réaliste», dans Répertoire II, Éditions de Minuit, Paris, 1964), en ceci que Couleurs croisées constitue la dramatisation musicale de certains des problèmes qui préoccupent la conscience morale et sociopolitique d'Henri Pousseur. L'autre œuvre est un collage magistral qui a été pensé comme l'illustration musicale d'une polémique engagée par le compositeur avec ses collègues et amis de l'avant-garde à la fin des années 1960 sur la question de l'ouverture de la pensée sérielle à une véritable généralisation, notamment sur le plan harmonique (cf. notre article : «La seconde apothéose de Rameau : au-delà de la polémique» dans Circuit, vol. 12, nº 1).

Si les notes du livret (quadrilingue!) de ce disque, rédigées par Robert Wangermée, tracent un très bon résumé du parcours général d'Henri Pousseur comme compositeur, l'introduction consacrée à chacune des deux pièces est malheureusement plutôt brève. Ainsi, on ne reprend pas le texte, succinct mais très clair à mon avis, que Pousseur lui-même avait écrit pour présenter Couleurs croisées dans le livret d'un disque Ricercar (RIC 036015) datant de 
1986 , et dont l'enregistrement présenté ici est en fait un repiquage «remasterisé» numériquement.

Couleurs croisées est une pièce pour grand orchestre composée en 1967 , commandée par la Koussevitzky Foundation in the Library of Congress et créée le 20 décembre 1968 à Bruxelles sous la direction de Pierre Boulez. Écrit comme une représentation de l'image que Pousseur se faisait des États-Unis - le pays de son commanditaire et de ses amis Cage, Tudor et al., du pop-art et du jazz - , image nourrie par un séjour personnel à Buffalo comme professeur et par les écrits de son ami Michel Butor sur l'Amérique, ce «poème symphonique » (cf. Pousseur, note du livret mentionné) veut « raconter la manière dont [lui] apparaissait "l'histoire" de ce pays et celle du "rapport entre les groupes sociaux qui le constituent" ", frappé qu'était le compositeur "par la situation ethnique (et d'ailleurs aussi économique) à la fois très bigarrée et très déchirée, vivante et conflictuelle dont ce grand pays, à quelque titre un résumé [...] de toute notre planète, est le théâtre.»

Pousseur construit la «courbe narrative très accusée [de l'œuvre] (les préparatifs d'un combat, celui-ci et ses arrière-faits ou amortissements) » à même une matière mélodique et harmonique tout entière déduite d'une harmonisation du chant We shall overcome, qui fut maintes fois entonné et «qui portait les foules lors des marches anti-ségrégationnistes des années 1960».

Eh bien, quelle expérience! Couleurs croisées nous fait voyager entre des monodies, des antiphonies, des homophonies et des polyphonies qui sont partagées entre groupes timbraux et familles instrumentales en constant renouvellement. Nous oscillons entre le simple et l'extrêmement touffu, entre des fragments très reconnaissables du chant cité et de larges mélodies plus ou moins disjointes qui en sont d'impressionnantes élaborations (extrême importance du plan mélodique!). Nous évoluons depuis les harmonies rugueuses du début (préparatifs), à travers les formidables étagements de tierces du point culminant central (combat) jusqu'aux quintes, quartes, sixtes et octaves dépouillées de la fin (espérances), harmonies donc en grande partie responsables et porteuses de la charge expressive de la forme. L'Orchestre philharmonique de Liège sous la direction de Pierre Bartholomée, ami et complice de longue date de Pousseur, donne de cette œuvre de presque 28 minutes, aux difficultés nombreuses (les problèmes de succession de tempi, de clarification des textures polyphoniques sont énormes), une lecture claire, précise, coup de poing, convaincante et convaincue, sentie et comprise.

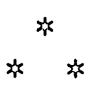


La seconde apothéose de Rameau pour 21 instruments a été composée en 1981 pour l'Ensemble InterContemporain et créée le 9 novembre de la même année par ce groupe sous la direction de Pierre Bartholomée. C'est ce dernier que l'on retrouve ici à la tête de l'ensemble belge Musiques Nouvelles.

Collage de citations plus ou moins transformées de (ou d'allusions à) Rameau, Schütz, Debussy, Stravinsky, Schœnberg, Webern, Boulez, Stockhausen, Berio et Bartholomée, et également de Pousseur lui-même (du Prologue dans le ciel tiré de Votre Faust et de Vue sur les jardins interdits), cette œuvre est cependant plus que la somme de ses emprunts. Je me suis déjà attardé longuement sur elle et l'ai analysée de façon assez détaillée sur plusieurs plans, y compris sa sémantique; on se référera à l'article précité, dont on pourra maintenant (enfin!) avoir le plaisir de pouvoir lire les longs tableaux formels en les confrontant avec la réalité musicale proposée par ce disque (pourquoi pas? à défaut de partition...).

J'ai entendu plusieurs enregistrements d'exécutions en concert de La seconde apothéose de Rameau (gracieusement prêtés par le compositeur), de même que la belle interprétation qu'en ont faite les étudiants du Conservatoire de musique de Montréal sous la direction de Véronique Lacroix, lors de l'événement Visages d'Henri Pousseur en janvier 2001. Il était temps qu'un enregistrement discographique fait en studio vienne permettre la pleine expression de cette œuvre, dont les défis techniques et musicaux sont également énormes. La fragmentation du discours qui naît de la juxtaposition rapide de citations et d'autocitations occasionne une succession abrupte de tempi, d'atmosphères ou de caractères qui demande virtuosité d'ensemble, intelligence, clarté de vision autant de la part du chef que des musiciens, cela pour que l'œuvre respire à l'aise et laisse percevoir son unité et sa courbe dramatique. Celles-ci sont d'un tout autre ordre que dans Couleurs croisées, car il s'agit plutôt, pourrait-on dire, d'une "comédie dramatique de la Culture » à laquelle il faut, pour être convaincante, non pas froideur et pure discursivité mais sensualité, énergie, attention aux couleurs et aux contrastes, etc. Et bien sûr, pour y atteindre, il faut le temps de travail et de préparation idoine...

Or ici sont au rendez-vous : clarté mélodique et rythmique, même dans cette réverbération assez généreuse (donc, idéal pour entendre certaines déductions mélodiques, comme ces petites formules bouleziennes qui sont partout!); attention aux détails d'articulation (pour alterner légèretés et appuis); fusions et couleurs instrumentales impressionnantes; et aussi, justesse dans le rendu des affects véhiculés par les harmonies. Partition en mains, j'ai redécouvert de nombreux passages qui m'ont fait apprécier tout le travail d'orchestration et de recomposition auquel s'est livré Pousseur. Par exemple, quand on connaît la version originale un peu sèche du petit Prologue dans le ciel tiré de Votre 
Faust, la version "complexifiée » qu'il en a élaborée pour cette Apothéose (et dont les fragments sont disséminés tout au long de cette dernière) relève du pur délire improvisateur, d'une débordante virtuosité imaginative. Et pareillement pour le travail autour de Vue sur les jardins interdits. Il résulte de tout cela des élans, des suspensions, des éclats et des chocs autant que des tendresses, des clins d'œil et des souffles remarquables. Discussion polémique à l'issue étonnante! On excuse ainsi le piano agréablement faux lorsqu'il fait entendre la valse schœnbergienne, ou les petits ratés individuels, car voilà une introduction éloquente à une œuvre riche et gratifiante, disponible maintenant pour le mélomane qui veut y entrer et y revenir.

$$
\text { ix }
$$

Croisement de couleurs harmoniques et de timbres, «mais aussi - et peut-être surtout - [croisements] de couleurs de peaux et de langues, d'idéologies et de cultures, voire de sentiments et d'espérances» (Pousseur, notes citées); polémique musicale généreuse comme un banquet et dont on n'a pas fini d'épuiser les leçons prospectives, musicales autant que morales : ai-je besoin de dire que Couleurs croisées et La seconde apothéose de Rameau m'émeuvent profondément, chacune dans leur registre, et que je les range dans mon coffre aux trésors personnel, chefs-d'œuvre ou monuments de la musique de cette génération de la seconde moitié du xx siècle, aux côtés de la Sinfonia de Berio et des Momente de Stockhausen, entre autres.

À défaut d'entendre et de réentendre en concert, données par des musiciens enthousiastes et convaincus, des œuvres riches, complexes, expressives comme celles dont il est question ici, parce que l'argent, l'audace, le temps de travail manquent aux sociétés de concert, aux orchestres symphoniques engoncés dans le passé et les problèmes budgétaires, aux radios publiques que l'on force à se plier aux diktats des cotes d'écoute, il reste le disque, la fréquentation personnelle et, au bout du don de votre temps, la conversion et l'adhésion, ce que je vous souhaite. 